ENCYCLOPEDDE Encyclopédie berbère

BERBERE

$35 \mid 2013$

35| Oasitae - Ortaïas

\title{
Onomastique libyco-berbère (Anthroponymie)
}

Salem Chaker

\section{OpenEdition}

Journals

Édition électronique

URL : https://journals.openedition.org/encyclopedieberbere/2816

DOI : 10.4000/encyclopedieberbere.2816

ISSN : 2262-7197

\section{Éditeur}

Peeters Publishers

\section{Édition imprimée}

Date de publication : 1 juin 2013

Pagination : $5760-5779$

ISBN : 978-2-7584-0184-1

ISSN : 1015-7344

\section{Référence électronique}

Salem Chaker, "Onomastique libyco-berbère (Anthroponymie) », Encyclopédie berbère [En ligne], 35

2013, document 017, mis en ligne le 12 mars 2021, consulté le 17 février 2022. URL : http://

journals.openedition.org/encyclopedieberbere/2816; DOI : https://doi.org/10.4000/

encyclopedieberbere.2816

Ce document a été généré automatiquement le 17 février 2022.

(c) Tous droits réservés 


\title{
Onomastique libyco-berbère (Anthroponymie)
}

\author{
Salem Chaker
}

1 Les différents domaines de l'onomastique libyco-berbère (anthroponomie, toponymie, ethnonymie, hydronymie, oronymie...), malgré d'assez nombreuses études et quelques travaux d'importance sur certaines périodes historiques (notamment ceux de J.-M. Lassère pour l'Antiquité), ne bénéficient pas de grands outils systématiques globaux. On ne dispose pas encore d'inventaires systématiques comme il en existe pour d'autres champs culturels de l'Afrique du Nord, notamment les domaines punique et latin (Benz, Jongeling, Lassère...). Le travail a été engagé par L. Galand pour la toponymie (1950), puis G. Camps pour l'anthroponymie (1993), mais il reste un chantier ouvert.

2 De plus, les études onomastiques restent assez fragmentées entre les diverses périodes historiques et disciplines. C'est évidemment que la tâche est immense : le corpus est d'une ampleur considérable. Le fonds onomastique berbère couvre un territoire gigantesque, fortement diversifié, et toutes les populations "indigènes" - de l'individu jusqu'aux groupes de tous niveaux (des familles élargies aux grandes confédérations tribales et aux ensembles régionaux) -, et ceci à travers toutes les époques historiques pour lesquelles on dispose de données, i.e. de la protohistoire à la période contemporaine. Le chantier est donc quasi infini si l'on considère notamment la microtoponymie qui commence tout juste à faire l'objet d'études approfondies (par ex.: Ahmed-Zaïd 1997).

3 L'intérêt des travaux onomastiques est pourtant grand pour la connaissance de l'histoire de la langue berbère, pour l'histoire culturelle et l'histoire tout court de l'Afrique Nord, à toutes époques.

4 Au plan de l'histoire, ces matériaux onomastiques apportent souvent des informations sociolinguistiques et socioculturelles précieuses: sur les langues en présence, sur les relations existant entre les différentes sociétés et cultures présentes en Afrique du Nord, sur l'histoire du peuplement, sur les mouvements de populations. Ils constituent donc un auxiliaire important des sciences historiques, d'autant qu'il n'existe quasiment 
pas de sources indigènes à l'histoire des Berbères, qui a toujours été écrite par le conquérant-dominant du moment.

5 Au cours de son histoire, le berbère n'a jamais atteint le statut de langue écrite stabilisée. Il n'y a donc pas (ou très peu) de sources littéraires anciennes qui permettent de suivre l'évolution de la langue. On comprend alors combien les informations linguistiques que fournit l'onomastique libyco-berbère peuvent constituer des données intéressantes ; elles permettent de vérifier ou d'infirmer les hypothèses reconstructives et d'éclairer tant soit peu les "siècles obscurs" de l'histoire de la langue. Ces traces sont les auxiliaires d'une difficile remontée dans le temps et, de proche en proche, elles peuvent contribuer à construire une image un peu plus précise des structures grammaticales, phonologiques et lexicales du berbère ancien.

Indépendamment de la masse de données à traiter, l'exploitation des matériaux onomastiques présente deux difficultés spécifiques :

- De par leur fonction de dénomination qui implique stabilité et continuité - notamment pour la toponymie -, la plupart des domaines de l'onomastique sont volontiers conservateurs, voire archaïsants, en ce sens qu'ils conservent souvent des matériaux lexicaux, des significations, des marques grammaticales anciennes, qui peuvent avoir totalement disparu dans les formes connues ou connaissables de la langue. L'analyse linguistique, formelle et sémantique, des matériaux est donc souvent délicate.

- L'histoire de l'Afrique du Nord est complexe et mouvementée, marquée par une succession de conquêtes qui ont pour beaucoup d'entre elles induit la présence directe et durable de langues extérieures (punique, latin, arabe, français...) : leurs traces dans l'onomastique nordafricaine sont considérables et il n'est pas toujours aisé de faire le départ entre les unes et les autres. Il y a un enchevêtrement des données, une sédimentation sur trois millénaires, souvent impressionnante: derrière des formes parfaitement berbères (ou arabes) contemporaines se cachent souvent des dénominations latines ou puniques. Certains exemples de toponymie sont remarquables : l'actuelle ville algérienne de Skikda (Philippeville durant la période française), s'appelait au Moyen âge Tasigda, forme parfaitement berbère, mais qui provient en réalité d'un Rusicade latin, qui repose lui-même sur un original punique Roš Ikade... Et dans l'usage kabyle actuel, les villes de Aïn El-Hammam et de Draa Ben Khedda sont appelées par tous Mišli (<Michelet) et Mirabu (<Mirabeau)...

7 Ces derniers exemples soulignent incidemment une autre source de difficulté dans l'interprétation socioculturelle ou sociopolitique des matériaux onomastiques: la fréquence des phénomènes de "dédoublement onomastique", à toutes époques et pour tous les domaines de l'onomastique. Des lieux, des individus, des rivières, des Dieux..., à époque antique, médiévale ou contemporaine, peuvent avoir deux, voire trois, noms distincts. Un exemple fameux est fourni par la stèle bilingue RIL 146 où le même personnage s'appelle C(aius) I(ulius) G(aetulus) en latin et KṬH W MSLWT en libyque. Dans l'Afrique du Nord contemporaine, peu de Berbères portent un nom patronymique officiel conforme à leur nom de famille traditionnel, encore tout à fait usuel lorsqu'ils s'expriment en berbère en milieu rural. En dehors de régions périphériques, surtout sahariennes, où il est souvent postérieur aux indépendances, l'état-civil actuel a été établi par l'administration française, puis reconduit par les Etats indépendants, sans qu'il ait été tenu compte, dans la majorité des cas, des usages traditionnels locaux. Deux exemples entre mille: les "Chaker" du village d'Adeni (Aït Iraten, Grande Kabylie) s'appellent en kabyle : Ijlili(ten). Les "Debiane" du village voisin d'Azouza s'appellent en kabyle Izwawen... Les officiers français qui ont établi l'état-civil à la fin du XIX ${ }^{e}$ ont très 
souvent attribué des noms de façon totalement arbitraire; parfois en ayant comme seule logique l'ordre alphabétique, ce qui explique des séries de noms de familles ubuesques comme celle du village d'Azouza : Aba, Ababa, Ababou, Abada, Abane..., dont aucun ne correspond à l'appellation traditionnelle locale. Fréquemment aussi, les noms ont été arabisés, parce que les officiers français étaient arabisants ou avaient des interprètes arabisants : un grand nombre des Ayt $X$ sont devenu des Ben $X$ ou Beni $X$ (par ex. les Ayt Ugugam sont devenu des "Ben Gougam"), parfois avec dédoublement au sein de la même famille qui se retrouve divisée en deux branches: Ayt X et Ben X (par exemple "Ait Kaci" et "Ben Kaci")...

8 Pareillement, les noms de lieux, grands ou petits, de villes comme de rivières, peuvent être (ou avoir été) totalement ou partiellement différents selon que l'on s'exprime (ou s'exprimait) en berbère, en arabe, en français ou, à date ancienne, en libyque, en latin ou en punique.

9 De plus, bien des toponymes latins ou arabes peuvent n'avoir été que des traductions d'appellations berbères antérieures qui ont continué à être en usage tant que la communication s'est faite en berbère. Et comme les sources écrites sont le plus souvent celles de la puissance et de la langue dominantes du moment, on peut vite être amené à tirer des conclusions erronées quant au degré de "punicisation", "romanisation", "arabisation", "francisation"... des populations locales et du territoire. Un bel exemple est fourni, près d'Alger sur la route de la Kabylie, par la petite ville de Thenia (nomenclature officielle algérienne), qui s'appelait Menerville durant la période française; les Kabyles la nomment Tizi n At Eiša ("Col des Aït Aïcha»), et les arabophones, dans l'usage local traditionnel: Thenia Beni Aïcha, qui est la stricte traduction de l'appellation kabyle.

10 On n'oubliera donc jamais qu'en Afrique du Nord un nom peut en cacher un autre, voire plusieurs autres!

11 Certains champs de l'onomastique présentent des difficultés spécifiques liées aux pratiques locales de dénomination: en hydronymie, la quasi totalité des noms répertoriés de fleuves, rivières, oueds divers, et ce à toutes époques, sont ceux qui ont été donnés par la puissance administrante. Dans l'usage traditionnel berbère, peu de cours d'eau ont un nom stable et commun sur tout leur cours; souvent, ils sont dénommés par le nom de la tribu dont ils traversent le territoire, changeant ainsi de nom en fonction de la localisation. Les hydronymes, anciens et actuels de nos cartes, ne sont donc que les dénominations que l'administration du moment leur a attribuées en sélectionnant, le plus souvent arbitrairement, parmi des usages locaux diversifiés.

12 Enfin dernier obstacle et non des moindres, pour les périodes anciennes comme pour la période contemporaine, les matériaux onomastiques berbères nous sont accessibles le plus souvent à travers le(s) filtre(s) de langues dominantes étrangères - punique, grec, latin, arabe, français - et non par une connaissance directe des sources berbères. En dehors du corpus libyque (avec toutes ses imprécisions graphiques intrinsèques), des rares sources berbères médiévales (ibadites, almohades) et des enquêtes de terrain et travaux contemporains encore bien rares (Foucauld, Laoust...), l'essentiel du matériel onomastique berbère connu est soumis au retraitement phonétique/orthographique, souvent très lourd, des langues et nomenclatures relais. Même les notations arabes médiévales, que l'on peut globalement créditer d'une meilleure transcription des matériaux berbères que le latin, présentent d'importantes transformations phonétiques/graphiques, qui elles mêmes sont souvent réinterprétées et opacifiées par 
les traducteurs-transcripteurs européens: ainsi une forme notée dans les sources arabes [yṣlītăn] (El-Bekrî, Ibn Khaldoûn) est retranscrite par le traducteur français (Mac Guckin de Slane) comme Isliten, alors qu'elle provient, sans le moindre doute possible, d'un berbère yəzlitən ( $<y$-zly tən; cf. infra) : le / $\mathrm{z} / \mathrm{du}$ berbère est devenu, comme attendu, un /ș/ en arabe puis un /s/ en français! Cette double distorsion phono-graphique n'est pas du tout anodine car elle peut orienter vers des analyses et interprétations totalement erronées : la racine berbère ZLY (" séparer, mettre à part ») n'a strictement rien à voir avec SLY («cailler, cuire rapidement à l'eau, ébouillanter »). Sur ce problème général, on se reportera à notre mise au point dans la notice M06b, EB XXX, 2010).

On voit que le corpus est à la fois composite, immense et rempli de distorsions historiques et linguistiques qui instaurent une incertitude, un doute permanent sur sa représentativité. Il serait assez illusoire dans un tel contexte de penser que l'onomastique d'une région aussi vaste et aussi tourmentée que l'aire berbère puisse être facilement analysable et interprétable. Les difficultés sont multiples et les éclairages éventuels toujours partiels et souvent hypothétiques. Mais c'est certainement le cas de la plupart des corpus onomastiques dans l'ensemble du monde.

\section{Anthroponymie libyco-berbère}

La première des constantes de l'anthroponymie libyco-berbère réside dans le système même d'identification des individus, caractéristique d'une société segmentaire, tribale et à tropisme patrilinéaire. Il est fondé sur :

- La filiation : $\mathbf{X} \boldsymbol{w}$ [fils de $]^{1} \mathrm{Y}$ (nom du père). Les cas de filiation matrilinéaire ne sont pas inexistants mais ils sont très rares.

-Et l'appartenance à des groupes sociaux emboîtés de plus en plus larges : famille élargie, tribu, ensemble confédéral... L'actualisation de l'appartenance de groupe dépendra de la situation concrète d'énonciation et du degré de précision dont on a besoin pour une identification pertinente.

Si l'on est en situation de proximité, à l'intérieur de la famille élargie, ou s'il s'agit d'un personnage connu de tous, le nom de l'individu suivi de celui de son père, éventuellement de son grand-père, suffira à l'identification, selon la séquence libyque et berbère :

$\mathrm{X} w / \mathrm{u}$ (fils de) Y $(w \mathrm{Z})$ :

MSNSN W GYY (W ZLLSN) = Massinissa fils de Gaïa (fils de Zilalsan)

Buluggin $w$ Ziri w Mennad

(šsix) Muhend $w$ Lhusin $=($ Cheikh $)=$ Mohand ou El-Hocine (saint kabyle $)$

Muhend $u$ Asmer (u Salem) = Mohand fils de Amar (fils de Salem)

Si l'on est en situation plus ouverte, au niveau du village ou d'une tribu, on précisera le nom de famille élargie :

Muhend u Azli (n) Iheddušen = Mohand fils de Ali (des) Haddouche

Enfin, si l'horizon est encore plus large, on précisera la tribu d'appartenance, voire la confédération, selon la séquence suivante :

Nom + fils de + Nom du père + Groupe d'appartenance 1 (+ Groupe d'appartenance

2).

KṬH W MSWLT,... MSKR(H), ZRMM(H) (RIL 146)

Muhend $u$ Acli Ayirat = Mohand fils de Ali des Aït Iraten 
Muha u Hammu Azayy = Moha fils de Hammou le Zaïane

Muhammad (ben) Sa'id al-Jannâdî al-Zawâwî ; en kabyle :

Muhend (w) Ssazid Ajennad, Azwaw = Mohand (fils de) Saïd, des Aït Jennad, le Kabyle.

18 Pour les personnages ayant une certaine notoriété, l'identification se fera alors très souvent uniquement par leur nom et celui de leur tribu ou confédération :

Meqq ${ }^{w}$ ran Agawa (chanteur kabyle) = Mokrane (des) Igawawen (confédération de la

Kabylie du Djurdjura)

Qasi Ibudrarən (tambourinaire kabyle) = Kaci des Ibudraren (ou Ayt Boudrar) Fațima

Tabacemrant (chanteuse chleuh) $=$ Fatima des Ayt Baamran

19 Dans les sources arabes, de très nombreux personnages d'origine berbère sont qualifiés et connus uniquement par leur appartenance tribale ou confédérale, suffisante, dans le monde des lettrés, à leur identification, l'ethnonyme devenant leur patronyme : AlWartîlanî* (des Ayt Wartilan), al-Waghlîsı* (des Ayt Weghlis), Al-Geštûlî (des Igeštulen), AlGhûbrînî (des Ayt Ghubri), Aș-Șanhağî (Aẓnag), Az-Zawâwî (le Kabyle)... Pratique qui s'est d'ailleurs souvent généralisée à travers l'état-civil moderne dans certaines régions berbérophones marocaines oit le nom de la tribu est devenu systématiquement patronyme, le plus souvent à travers une forme arabisée: notre collègue et ami berbérisant M. Elmedlaoui est évidemment originaire de la tribu des Imədlawən.

Il s'agit donc d'un système de dénomination à " géométrie variable » en fonction du besoin précis d'identification, qui permet d'apporter les éléments de précision nécessaires et suffisants à chaque situation.

21 Sur le plan de la forme des anthroponymes, élargissant et systématisant le travail pionnier d'O. Rössler (1958), on a établi dans deux études déjà anciennes (Chaker 1972, 1985) les grandes lignes d'un modèle d'interprétation linguistique que nous reprenons ici. Ce modèle couvre aussi bien des données de l'anthroponymie actuelle (prénoms berbères encore usités par les berbérophones) que les matériaux provenant des périodes médiévale et antique. Il permet, à partir d'un nombre significatif d'exemples (plus de 150), de montrer qu'il existe dans l'anthroponymie des Berbères un noyau dur, permanent et interprétable dans le cadre des structures grammaticales fondamentales de la langue.

Il va de soi qu'un tel cadre, portant sur une période historique de plus de deux millénaires, ne saurait rendre compte de la totalité des anthroponymes berbères, ceci même si l'on avait les moyens d'écarter à coup sûr tous les emprunts à des langues et cultures allogènes.

Moyen d'identification sociale des individus, l'anthroponymie est un puissant marqueur d'identité culturelle et linguistique. A travers elle, il est possible d'avoir une idée de la vitalité, de la permanence, des reculs ou des avancées de l'identité berbère face aux autres cultures. Il n'est pas sans intérêt de constater que beaucoup des chefs de révolte contre l'autorité romaine porte des noms berbères; ou, à la fin du monde antique, après l'effondrement de l'autorité romaine, de voir (re)surgir, de façon massive, une anthroponymie libyco-berbère, y compris chez les princes et petits rois comme Masuna* qui se proclament « Rex gentium maurorum et romanorum »!

Bien sûr, les matériaux sur lesquels l'on peut s'appuyer dans ce domaine sont trop incertains, obscurs et fragmentaires pour qu'il soit permis d'en tirer des conclusions trop tranchées. Le problème de la représentativité (qualitative et quantitative) des matériaux est crucial et oblige à une grande prudence. Que peuvent en effet 
représenter les quelques dizaines de noms berbères antiques ou médiévaux analysables avec un minimum de certitude, au regard des multitudes d'anthroponymes à jamais oubliés, non fixés par le témoignage écrit ou rebelles à toute interprétation?

De même, toujours dans la perspective de l'utilisation du nom comme indice d'identité culturelle, on n'oubliera pas le risque permanent de distorsion lié au phénomène, sans doute très général, de "dédoublement anthroponymique" évoqué précédemment. Un nom peut en cacher un autre et l'identité "latine" ou "arabe" peut n'avoir été que très superficielle, voire même une simple traduction ou une façade officielle sans ancrage dans le vécu des populations locales.

La conquête arabe et l'introduction de l'islam ont sans aucun doute constitué une rupture profonde dans les usages onomastiques de l'Afrique du Nord. Très vite une masse d'anthroponymes musulmans font leur apparition et renouvellent profondément le stock des noms propres. Cependant, là encore, il convient de se méfier des effets de masquage: les noms qui nous sont transmis par les textes arabes sont ceux de personnages de premier plan, en contact direct avec les Arabes et l'islam (qu'il s'agisse de relations de conflit ou de collaboration). Ils ne sont vraisemblablement représentatifs que de milieux restreints. De plus, là aussi, un nom "officiel" (musulman) peut se surajouter à un autre (berbère). Les exemples précis de baptêmes islamiques tardifs ne sont du reste pas rares.

Il convient aussi de ne pas sous-estimer la forte capacité d'intégration et de "naturalisation" de la langue berbère: des anthroponymes puniques, latins, arabomusulmans ont connu un processus de berbérisation très profond qui en a fait d'authentiques noms berbères, avec souvent même une forte valeur emblématique de "berbéritude" ; il existe des exemples contemporains fameux, d'autant plus marquants qu'ils sont largement usités et dans plusieurs régions berbérophones :

Muhend, Mhend issus de Mohammed, Hend, issu de Ahmed, Haddu...Tasazdit

(berbérisation de Sazdiya, "Heureuse")...

Bien que d'origine arabe, ils identifient immédiatement leur porteur comme berbère !

La même capacité d'intégration est décelable à époque ancienne : le corpus de bilingues du RIL fournit des équivalences berbères régulières de noms puniques :

\begin{tabular}{|l|l|}
\hline 'BDŠMN & $>$ libyque : ŠMN (RIL 2) \\
\hline 'BDRŠ & $>$ libyque : DRŠ (RIL 1, 2) \\
\hline$\ldots$ & \\
\hline
\end{tabular}

Et pourtant, malgré ces bouleversements profonds, ces pressions étrangères permanentes, si dans le corpus des anthroponymes "berbères", on écarte les matériaux manifestement étrangers (puniques, latins, arabes...), on constate une réelle permanence des formes et des pratiques.

31 Non seulement, ce noyau "berbère" de l'anthroponymie antique et médiévale, voire contemporaine s'intègre dans le même modèle d'explication linguistique, mais les formes elles-mêmes se retrouvent souvent identiques à travers les siècles. Cette permanence structurale et lexico-sémantique va même, dans quelques cas favorables, jusqu'à la période actuelle (cas de YDR / Iader / Yidir - Yedder...). Si l'on ne sépare pas 
l'anthroponymie de l'ethnonymie, qui est un véritable conservatoire d'anthroponymes puisque les noms de tribus réfèrent le plus souvent à un ancêtre commun, réel ou mythique, on peut même affirmer que la continuité est totale : toutes les formes de noms propres identifiables dès l'Antiquité se retrouvent dans les noms de tribus et de groupes actuels.

\section{Les différents types d'anthroponymes libyco-berbères}

Ce récapitulatif ne prétend pas épuiser les possibilités. Il ne regroupe que les types de formations suffisamment bien attestées pour que leur régularité même soit un gage de solidité de l'analyse proposée. On notera que les différents types identifiés n'ont rien de spécifique au domaine berbère, en dehors, bien sûr, des matériaux morphologiques et lexicaux utilisées: on retrouve des structures tout à fait similaires dans les aires culturelles voisines et/ou apparentées, notamment sémitique.

$\mathrm{Au}$ niveau sémantique, les anthroponymes berbères, de l'Antiquité jusqu'à la période contemporaine, lorsqu'ils sont interprétables, ont fréquemment une valeur :

34 - Prophylactique : il s'agit alors de détourner du porteur du nom les puissances du mal, la maladie, la mort... Pour cela on se réfère à des antidotes susceptibles de contrecarrer ou repousser ces forces négatives. Les cas les plus emblématiques et apparemment les plus stables depuis l'époque libyque, sont les noms qui désignent le noir/Noir, l'esclave: Sammag, Suggen / Seggen (= "il est noir"; A, MA), Akli, Taklit (= "Esclave noir(e)"; BC, kabyle). Les Noirs avaient traditionnellement, dans toutes les sociétés berbères, un statut social et/ou professionnel spécifique: artisans (forgerons), bouchers, cordonniers, musiciens... toutes activités sensées être en lien avec les forces chtoniennes, le feu. Dans les sociétés contemporaines, cette fonction prophylactique est encore parfaitement perçue et explicitée : en Kabylie, ce sont les femmes qui ont perdu successivement plusieurs enfants qui nomment leur dernier-né Akli ou Taklit, "Esclave noir(e)", afin de détourner la mort de lui. On raconte même (notation personnelle) l'histoire d'une femme qui, perdant régulièrement ses bébés, décida de nommer son dernier-né Izzzan, "Excréments" ; malgré cette précaution, l'enfant ne survécut pas et la malheureuse se mit à ce lamenter :

Ay Izzan a mmi, ur təddirəḍ, ur d-təğğid isəm yəlhan!

«Ô Excréments mon fils, ni tu as survécu, ni tu nous as laissé un joli nom ! »

- Propitiatoire: les exemples sont innombrables, dans le corpus libyque, médiéval et contemporain, de noms faisant référence aux qualités et vertus, aux succès, à la longévité souhaités à son porteur. YDR - Yidir, "qu'il vive!"; YRN - Yərna, "il a vaincu"; YZMR - Yəzmər, "Il est capable, il peut" : YFTN - Yiftən, "il les surpasse": MSTN - Amastan, "défenseur, protecteur"...

Les cas de noms d'animaux, motivés bien sûr par les vertus et qualités qui leurs sont prêtées, sont fréquents chez les Touaregs et semblent pouvoir être détectés dès l'Antiquité :

Bagga, Beggi, Abeggi, "Chacal”, Ilu, "Eléphant”, Amayas, "Guépard”... Pour les noms de femmes, on en trouve des traces au Moyen âge et en Kabylie contemporaine: Tasekkurt / Sekkura, "Perdrix", Tamilla, "Tourterelle"... 


\section{Nom verbal simple (forme verbale isolée)}

Il porte une marque spécifiquement verbale qui permet de l'identifier aisément. La plus fréquente est l'indice de la $3^{\mathrm{e}}$ personne du masculin singulier $y-$ (ou $\left.i-\right)$, "il -" (YZMR = "Il peut, il est capable, il endure, supporte"). On relève quelques exemples portant la marque du féminin correspondant $t-$, "elle - (TZMR = "Elle peut..."), qui doivent être des noms de femmes, évidemment beaucoup plus rares parmi les noms de personnages historiques.

C'est là le mode de formation le plus net, le plus fréquent et le plus stable puisqu'il est abondamment illustré dans les périodes libyque (cf. RIL) et médiévale et qu'il est encore représenté par des cas indiscutables, très largement répandus jusqu'à présent, parfaitement analysables : YDR, Iader, Yider, Yedder, Yidir “(qu') il vive/il est vivant".

$\mathrm{Au}$ niveau sémantique, ces formes puisent principalement dans des champs lexicaux valorisants, référant à des caractéristiques ou actions positives des individus et ils ont une fonction propitiatoire évidente :

\begin{tabular}{|l|l|}
\hline$-y$-idir & “(qu') il vive/il est vivant” \\
\hline$-y$-frn & “il est choisi, élu” \\
\hline$-y$-rna & “il a vaincu” \\
\hline$-y$-zmr & “il peut, il est capable” \\
\hline$-y$-zly & “il à part/unique...” \\
\hline
\end{tabular}

Du point de vue aspectuel, ces anthroponymes sont construits aussi bien sur des thèmes de prétérit (ou accompli), renvoyant à des procès ou qualités posés comme réalisés ( $y$-rna, "il a vaincu"), que sur des thèmes d'aoriste, à valeur optative ou injonctive : $y$-idir, “(qu) il vive !". Ce dernier cas, à travers les époques et les régions, semble avoir été construit aussi bien le thème d'aoriste (Yidir = «Qu'il vive ! ») que sur le thème de prétérit (Yəddər = «Il est vivant, il vit »).

41 Notons que plusieurs de ces formes verbales simples entrent dans la composition d'anthroponymes complexes à noyau verbal (y-rna $\rightarrow$ y-rna tən), ce qui est une confirmation à rebours de la validité de l'analyse proposée.

Les corpus libyque et médiéval en fournissent un nombre considérable; dans RIL, la majorité des noms à initiale $Y$ - (noté I dans le RIL) - on en compte près d'une centaine - et une partie de ceux à initiale $\mathrm{T}$ - doivent appartenir à ce type :

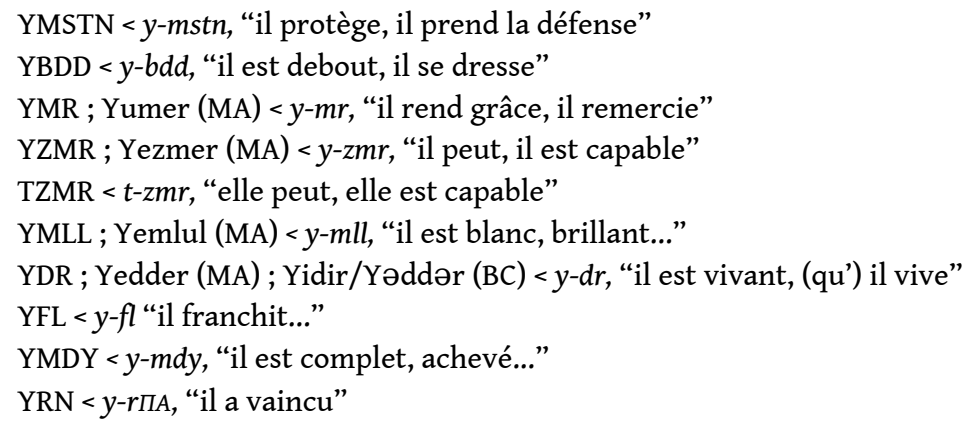


Hiempsal; Yemsal <y-msal ou, plus probablement, Yemșal <y-mzal

Iuba ; Yuba (T) $<y$-uba (?)

Yefren (MA, NPT) $<y$-frn "il est choisi, élu"

Yezly (MA) < y-zly "il est à part/unique..."

Yaymur (MA) < y-aymur "il a prospéré" (sans doute de la racine arabe $\varepsilon m r$ )

Yəqqur (T) < y-qqur "il est dur/sec"

Ethnonymie/ Toponymie :

Yefrək (Ayt Yefrek) $<y$-frok

Yemmel (Ayt Yemmel) <y-mməl

Yefren (At Yefren : Dj. Nefoussa*, $)<y$-fron

\section{finale pouvant être :}

- verbale : $-n$ "ils" = indice $3^{e}$ pers. masc. plur. ; $-n$, marque de participe* verbal ;

Certains verbes (verbes de qualités) ne portent pas de marque de personne explicite à la $3^{e}$ pers. masc. sing. du prétérit: ils ne sont pas immédiatement identifiables comme verbes, d'autant que les nominaux peuvent aussi ne porter aucune marque formelle apparente et peuvent donc facilement être confondus avec eux. Or, on peut penser que ce stock de verbes (référant à des qualités ou états stables) a été intensivement utilisé par l'anthroponymie. Il s'agit d'un type qui a dû être fréquent mais faiblement identifiable, surtout lorsque, comme en libyque, on ne dispose pas d'une représentation des voyelles et de la tension consonantique. De nombreux anthroponymes antiques et médiévaux semblent appartenir à cette catégorie :

Suggan, Suggen, Seggen (A, MA) < SGN (isgin) "e. noir" = "il est noir"

Sammac <Sammag (A, MA) < SMG "e. noir" (cf. isməg : "esclave noir")

Macur-(A) (<Maqqur) < MrR/MQR (imyur/imyar) "e. grand"

Mənnad (MA, BC) < MND (?). La forme kabyle actuelle, Mənnad, correspond

typiquement à une $3^{\mathrm{e}}$ pers. masc. sing. sur thème de prétérit d'un verbe de qualité

(cf. zəggay, "il est rouge")

....
47 ou

- nominale : $-n$, marque de pluriel ; $-(a) n=$ suffixe d'adjectif*.

analyse de ce type de formation est de ce fait souvent indécise. Dans quelques cas, lorsqu'il y a combinaison d'un préfixe $y-$ et du suffixe $-n(y-n)$, l'incertitude est pratiquement levée : il s'agit certainement d'un participe verbal (type fréquent dans l'Antiquité comme au Moyen Age) :

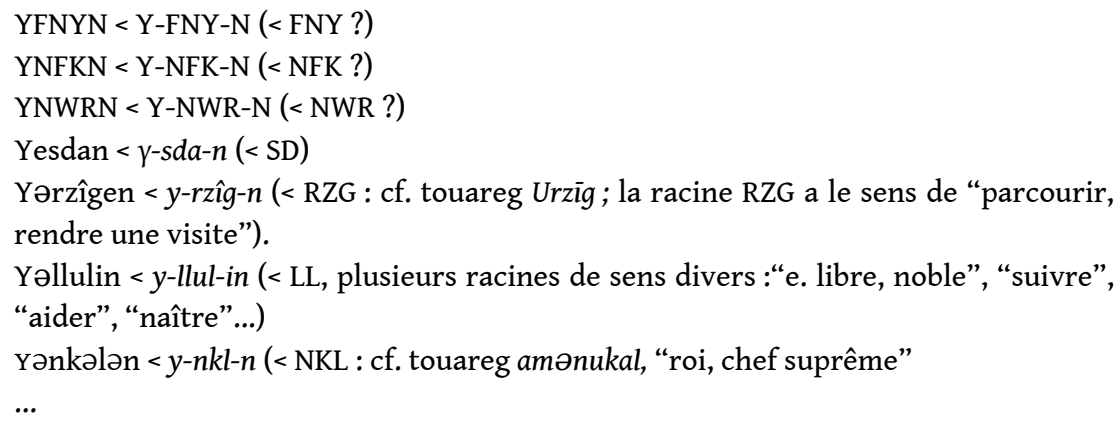




\section{Nom verbal complexe : Verbe + Pronom Personnel Régime} actuellement que dans l'ethnonymie. Plusieurs de ces formations sont communes aux temps libyques, médiévaux et modernes (YRNTN / Yernaten; YRTN / Yiratən). Le noyau verbal est le plus souvent une $3^{\mathrm{e}}$ personne de masculin singulier sur thème de prétérit ( $\mathrm{y}-$, "il-") et, l'on y retrouve très logiquement des formes connues comme nom verbal simple (y-rna, y-aymur, $y$-zly,..). Le complément pronominal est toujours un personnel pluriel, le plus souvent direct (TN =-tĂn,-tən, "les"), quelquefois indirect (SN = asăn, asən, "à eux"); le schéma le plus courant est donc celui d'un phrase verbale élémentaire :"il-verbe_les/à eux"

Le réfèrent du pronom régime est évidemment obscur, et sans doute variable selon le lexème verbal : TN/tən - "les" représente "les ennemis", "les rivaux", dans y-rna tən, "il les a vaincus", dans $y$-if tən, "il les surpasse" et dans $y$-ugur tən (Jughurtha*), "il est plus fort qu'eux"; mais peut-être les "Dieux" dans YRTN = $y$-ira tən, "il les aime" (dans Ayt Yiraten. confédération tribale kabyle) :

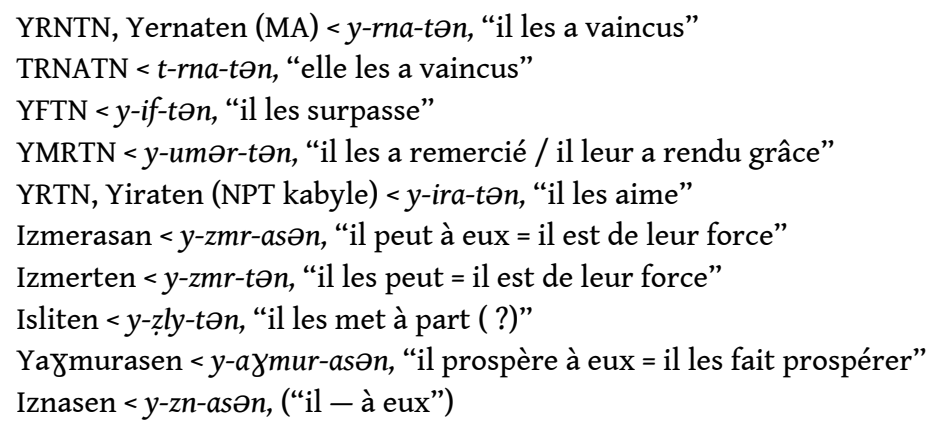

51 Yellilten < y-llil-tən (ethnonyme kabyle: At Yellilten), "il les -la racine LL a des significations diverses: "aider" (aləl), "ê. libre, noble" (lullət), "suivre" (ilal); on penchera pour cette dernière (touareg ilal/yəllil, "suivre"), morphologiquement plus compatible. On en relève le correspondant féminin en libyque :

TLLTN $<$ T-LL TN $={ }^{*}$ t-llil-tən, "elle les suit".

\section{Nominal simple (forme nominale isolée)}

a) Les noms à marques nominales apparentes, identiques à celles du nom commun actuel :

masc. : $a-/ /$ fém. : $t(a)-(t)$.

Il s'agit de substantifs (ou d'adjectifs) communs utilisés comme noms propres. Ce type, bien vivant dans l'anthroponymie berbère actuelle, est aussi abondamment représenté au Moyen âge. En revanche, au masculin, il est difficile à identifier dans le corpus antique du fait de la non-notation des voyelles en libyque ; le seul cas probable provient des sources latines: Aylima(s). En revanche, au féminin, on peut suspecter les nombreuses séquences T-T du corpus libyque d'appartenir à ce type; dans le seul RIL, on en relève une bonne quinzaine :

Libyque :

TDZT, TKDNT (cf. KDN, masc.), TKDT, TMDT (cf. Touareg: Timidit), TNGT, TMSWT(H), TNT, TSDT (cf. SD, masc. ?) TSLT (cf. SL, masc. ?), TRFNT, TRLT, TRẒT(H), TRNT(H) (cf. RN, masc. ?), TRSMT, TŠBLT, TŠKRT, TŠT(H)... 


\begin{tabular}{|c|c|c|c|}
\hline \multicolumn{4}{|l|}{ Moyen âge : } \\
\hline Masc. & & Fém. & \\
\hline Aggag & "Lettré" & Tasekkurt & "Perdrix" \\
\hline Agellid & “Roi” & Tiski & \\
\hline Anegmar & "Chasseur" & Tifawt & "Lumière, Matin" \\
\hline Amyar & "Chef' & & \\
\hline Iggig & "Eclair" & & \\
\hline Awray & "Jaune/Doré" & & \\
\hline Aderyal & "Mal-voyant/Aveugle" & & \\
\hline & $\ldots$ & & \\
\hline Touareg: & & & \\
\hline Amastan & "Défenseur, Protecteur" & Tabhawt & "Blonde/Rousse" \\
\hline Amyar & "Vieux/Chef" & Tamyart & "Vieille" \\
\hline Ilu & "Eléphant" & Timidit & "Amie, Compange" \\
\hline Amayas & "Guépard" & & \\
\hline Ebəggi & "Chacal" & Tebəggit & "Chacale" \\
\hline Aməllal & "Antilope addax" & & \\
\hline Amdəy & "Girafe" & & \\
\hline Berbère Nor & & & \\
\hline Akli & "Esclave noir" & Taklit & "Esclave noire" \\
\hline Aməzzyan & "Petit" & & \\
\hline Aməq $q^{w} r a n$ & "Grand" & & \\
\hline
\end{tabular}

b) Les noms propres sans marque: n'étant pas formellement marqués comme nominaux, leur identification n'est pas toujours aisée. Il s'agit de traces des époques où les marques du nom n'étaient pas encore obligatoires. Ce type est connu à l'heure actuelle : 
Məddur (ou Muddur), "vivant", alterne avec Ameddur,

Məqq" ${ }^{w}$ ran, "grand" (Mokrane), alterne avec Ameqq ${ }^{w}$ ran (Amokrane),

Məzzyan, "petit" (Meziane), avec Aməzzyan (Ameziane)....

Hingu, "Ennemi, adversaire", avec Ahəngu

Baǵga, Bəggi, "chacal” avec Ebəggi

Maksan - Maksən, "Ennemi, adversaire", avec Amaksan... plus difficile à repérer avec certitude dans la période antique, du moins au masculin, bien que l'on soit en droit, comme on l'a rappelé ci-dessus, de penser qu'il était fréquent.

\section{Nominaux complexes : plusieurs sous-types sont identifiés}

b) MS + Nom : ce type, bien attesté dans l'Antiquité et représenté par l'emblématique MSNSN-Massinissa, n'est apparemment plus du tout pratiqué au Moyen Age. Ces syntagmes peuvent recevoir deux analyses concurrentes :

- Il peut s'agir d'une séquence MS "Maître/Seigneur" (məss, măss, en touareg) + Nom ou Déterminant (= "Maître X"), parallèle aux formes arabes actuelles Sidi X;

- Ou de dérivés en $m s$ - de bases nominales, du type abrid "chemin" > amesbrid, "passant, voyageur...", adrar "montagne" > aməsdrar/ iməsdurar, "montagnard". Cette procédure de dérivation, qui reste très productive en touareg à partir de toponymes ou d'ethniques (> "celui de X", "celui des Y") :

aməssgərəs, "homme des Kel Geres", aməskano, "homme de Kano" pourrait expliquer une bonne partie de ces complexes antiques (MSDLN $-{ }^{*} m s-d l n$, "Celui de DLN").

c) MSTN + Nom :

Le premier élément est sans aucun doute le nom (a)mastan encore usité comme prénom chez les Touaregs et dont le sens est parfaitement connu : "protecteur, défenseur..." du verbe mesten "protéger, défendre (en paroles), combattre...". Il s'agissait peut-être dans l'Antiquité d'un véritable titre :

MSTNB = Mastan $(a) B(a)$, "Protecteur/Défenseur Aba", forme berbère du nom Mastanabal (cf. Chaker, M57b, EB XXXI, 2010). 
MSTNSN/MSTNZN = Mastan SN, à rapprocher des noms des rois Mastanesosus et Mastenizen. Peut-être *Mastan-SĂN, "Leur protecteur".

\section{Les complexes négatifs}

64 anthroponymes sont fréquents dans l'Antiquité et abondent littéralement au Moyen Age, mais surtout dans l'ethnonymie. On en retrouve quelques traces dans les pratiques anthroponymiques des Touaregs actuels, mais globalement, ils ne subsistent plus que dans les ethnonymes Ouriaghel < Wer yayel, tribu rifaine).

\section{des lacunes et incertitudes dans la notation des voyelles.}

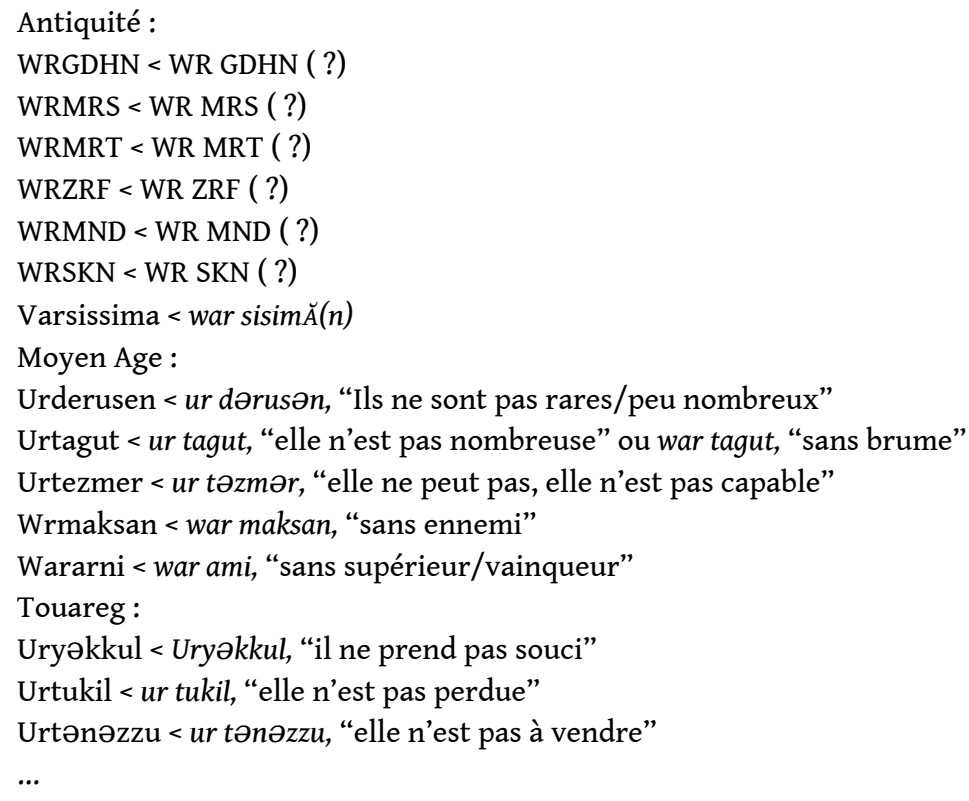

\section{Les complexes Support + Déterminant}

Il s'agit d'un support de détermination (pronom démonstratif, défini ou indéfini ; cf. Galand 1974) : wa, (w)i /... “celui”, ta, ti... "celle”, suivi d'une détermination, nominale ou verbale :

wa (n) + Nom (= "celui de X") : wa + Verbe (= "celui (qui) X").

Ces formes sont très nombreuses au Moyen Age et foisonnent dans l'onomastique touarègue actuelle (anthroponymie et toponymie) où elles fonctionnent en fait plutôt comme des surnoms. Aucun cas n'a pu être identifié avec certitude pour la période antique.

Waggutan < wa ggutĂn, "Celui (qui est) abondant"

Wigelden < wi gəldən, "Qui étant roi" 
Wiftin < wi ftin, "Qui se multplie, prolifère"

Widdaren < wi ddarən, "Qui est vivant"

Wasyagut < wa s yagut, "Celui avec (lequel) il abonde"

Wanzemmar < wa n zəmmar, "Celui de Zemmar"

Tinizammaren < ti n izammarən, "Celle des agneaux/béliers"

Touareg:

Tatŭfət < ta tufət, "celle qui est supérieure"

WanĂləlli < wa n Ăləlli, "celui de libre/noble = le noble"

Imməgārən < i n məgārən, "Un des hôtes"

Taməllət < ta mellet, "celle étant blanche = la blanche"

TintĂməlla < ti n tĂməlla, "celle de la grâce"

Wadyusən < Wa d-уusəп, "celui qui est arrivé"

$\cdots$

L'application du modèle d'interprétation linguistique sur des matériaux assez abondants provenant de l'Antiquité (surtout de RIL), de la période médiévale (EI-Bekrî et Ibn Khaldoûn) et des usages contemporains,

- Confirme la validité du modèle lui-même puisqu'il rend compte de formes nombreuses, réparties sur toutes les périodes historiques ;

- Met en évidence la grande stabilité du système anthroponymique berbère et l'absence de rupture radicale dans ce domaine entre l'Antiquité et le Moyen Age ;

- Fait cependant apparaître certaines évolutions et spécificités distinguant les deux époques : si les formes de base sont bien communes à l'Antiquité et au Moyen Age, on voit cependant se dessiner des changements dans l'équilibre numérique des divers types (certains régressent, d'autres deviennent envahissants) et, par ailleurs, on voit apparaître avec le temps certaines constructions complexes secondaires qui connaissent encore aujourd'hui un grand succès dans les usages de certains groupes berbérophones.

De manière plus précise, les évolutions décelables depuis l'Antiquité sont les suivantes :

a. Une sensible régression des formes verbales et nominales complexes, du moins pour ce qui est de l'anthroponymie vivante de chaque époque ;

b. Une nette augmentation des formes nominales à marques de substantif explicites ( $a$-et $t-$ t);

c. Une tendance au figement des complexes négatifs qui apparaissent surtout dans des ethnonymes au Moyen Age ;

d. L'apparition massive à partir des temps médiévaux des séquences "Support + détermination nominale ou verbale" (“Celui de X"/“Celui qui Y”), qui sont typiquement des surnoms plutôt que des prénoms.

Et, bien, sûr, une irruption massive, voire une véritable submersion à partir de la conquête arabe et de l'islamisation, des anthroponymes arabo-musulmans. Tendance fortement combattue depuis quelques décennies par un retour en force des prénoms berbères anciens dans la plupart des groupes berbérophones. Le succès de l'Officiel des prénoms berbères de Kamal Nait-Zerrad atteste de la vigueur de ce mouvement de reberbérisation militante. 


\section{Conclusion}

Dans le véritable maelström culturel qu'a été l'histoire de l'Afrique du Nord au cours des trois derniers millénaires, l'onomastique, et particulièrement l'anthroponymie libyco-berbère, présente, malgré les brassages, voire les submersions culturelles et religieuses, des éléments de continuité indéniables, même si, bien sûr on ne peut nier ou minimiser les apports et influences allogènes, puniques, latines, chrétiennes, et surtout arabo-islamiques. Il s'est, malgré ce contexte mouvementé, maintenu à toutes époques un noyau anthroponymique libyco-berbère stable, qui pour une part nonnégligeable peut s'analyser de manière quasi immédiate à partir des structures élémentaires de la grammaire et du stock lexical fondamental de la langue berbère.

L'anthroponymie, et plus largement l'onomastique, constitue manifestement un des éléments de la " permanence berbère » de l'Afrique du Nord, chère à Gabriel Camps.

$\mathrm{Au}$ plan anthropologique, cette continuité doit être interprétée comme l'indice de la persistance d'une conscience identitaire collective « berbère » face ou à côté des autres identités ethno-culturelles présentes sur le territoire.

$76 \mathrm{Au}$ plan linguistique, elle met en évidence une grande stabilité des structures élémentaires de la grammaire berbère - en tout cas de la morphologie verbo-nominale - et du matériel lexical fondamental de la langue. Certaines données sont d'ailleurs plus que des indices ou hypothèses et peuvent être considérées comme des certitudes : des paires (nominales) comme KDN / TKDNT ou (verbale) YRN / YRNTN et TRNTN, YDR/ Iader/Yidir ne laissent quasiment aucune place au doute.

Ce qui soulève incidemment un problème théorique considérable et troublant : celui de la vitesse d'évolution de la langue berbère, qui apparaît singulièrement lente en comparaison avec ce que l'on sait d'autres domaines linguistiques voisins. Car si ces analyse sont exactes, elles impliquent que les lignes essentielles de la morphologie verbo-nominale du berbère sont à peu près les mêmes depuis au moins deux millénaires et que la langue, sur ce plan, a peu évolué. Le berbère serait donc une langue à évolution très lente, ce qui rejoindrait l'intuition de certains savants du $\mathrm{XIX}^{\mathrm{e}}$ siècle, comme Renan à propos des langues sémitiques... Mais c'est là un vaste débat qui dépasse le cadre de cette notice (cf. 026 « Origine(s) berbères : linguistique et préhistoire »).

\section{Abréviations}

\begin{tabular}{|l|l|}
\hline A & $:$ Antiquité \\
\hline MA & $:$ Moyen Age \\
\hline BC & $:$ Berbère contemporain \\
\hline T & $:$ touareg \\
\hline NPT & $:$ Nom propre de tribu (ethnonymes) \\
\hline RIL & $\begin{array}{l}: \text { Recueil des Inscriptions Libyques, voir bibliographie, J.B. Chabot. } \\
\text { Les formes issues du RIL sont toujours données en majuscules. }\end{array}$ \\
\hline
\end{tabular}


$\rightarrow$ Voir aussi : « Maces et autres noms... : mise au point linguistique » (M06b) ; « Nom » (N62) et « Nomination» (N68) « Toponymie».

\section{BIBLIOGRAPHIE}

[Les références susceptibles de présenter un intérêt du point de vue de l'onomastique libycoberbère sont innombrables : travaux de géographes, historiens, ethnologues, linguistes... On ne signale ici que les travaux importants consacrés spécifiquement à l'onomastique ainsi que les principales sources de données.]

AHMED-ZAïD M., 1997 - Contribution à l'étude de la toponymie villageoise kabyle, Thèse de Doctorat, INALCO, 1997 (dir. : S. Chaker), 681 p. + 21 p. + XVII p..

BASSET A., 1948 - « Sur la toponymie berbère et spécialement sur la toponymie chaouïa Aït Frah ", Onomastica. Repris dans Articles de dialectologie berbère, Paris, Klincksieck, 1959, p. 163-166.

BASSET A., 1950 - Pour une collecte des noms propres, Bull. IFAN, XII, p. 535-539.

BEGUINOT F., 1930 - "Per gli studi di toponomastica libico-berbera”, Congr. Geogr. Ital., 3, Naples.

BENRAMDANE F. (Coord.), 2005 - Des noms et des noms... Etat-civil et anthroponymie en Algérie, Oran, Editions du CRASC.

BENRAMDANE F. \& ATOUL B. (Coord.), 2005 - Toponymie et anthroponymie de l'Algérie. Recueil bibliographique général, Oran, Editions du CRASC.

BENRAMDANE F. \& ATOUL B. (Coord.), 2005 - Nomination et dénomination des noms de lieux, de tribus et de personnes en Algérie, Oran, Editions du CRASC.

BENZ F., 1972 - Personal Names in the Phoenician and Punic Inscriptions, Rome, Biblical Institute Press.

CAMPS G., 1960 - Massinissa ou les débuts de l'Histoire (Libyca, Archéologie-Epigraphie, VIII).

CAMPS G., 1993 - « Liste onomastique libyque d'après les sources latines », REPPAL, VII-VIII, p. 39-73.

СНАВот J.-B., 1940/1941 - Recueil des inscriptions libyques, Paris, Imprimerie Nationale.

CHAKER S., 1972 - « Onomastique libyco berbère », Encyclopédie Berbère (édition provisoire, 1 (Aix, LAPMO), $9 \mathrm{p}$.

CHAKER S., 1983 - « La langue berbère à travers les textes anciens : El-Bekri », ROMM, 34/1. Repris dans : Linguistique berbère : études de syntaxe et de diachronie, Paris/Louvain, Peeters, 1995, chap. 12. CHAKER S., 1985 - « Onomastique berbère ancienne (Antiquité/Moyen âge) : rupture et continuité, BCTH, n.s., 19, 1983 [1985] ( = 2 Congrès d'Histoire et d'Archéologie de l'Afrique du Nord), p. 483-497. Repris dans Textes en linguistique berbère, Paris, Editions du CNRS, 1984, et Alger, Bouchène, 1991, chap. 14).

CHAKER S., 1995 - Linguistique berbère. Etudes de syntaxe et de diachronie, Paris/Louvain, Peeters, 1995, 275 p. Edition algérienne augmentée parue sous le titre : Manuel de linguistique berbère II, Alger, ENAG, 1996, 289 p. 
CHAKER S., 2010 - «M06b. Maces et autres noms propres anciens : mise au point linguistique », EB, XXX, p. 4463-4465.

CHERIGUEN F., 1993 - Toponymie algérienne des lieux habités. Les noms composés, Alger, Epigraphe.

EL-BEKRî, 1965 - Description de l'Afrique septentrionale (trad. Mac Guckin de Slane), Paris, Maisonneuve (texte bilingue).

FOUCAULD Ch. (de), 1940 - Dictionnaire abrégé touareg-français des noms propres, Paris, Larose.

GALAND L., 1947 - « Baquates et Bargaawâta », Communication au $2^{\mathrm{e}}$ congrès international de toponymie et d'anthroponymie (Paris, 1947), Hespéris, 1948.

GALAND L., 1948 - «Eléments non radicaux dans les toponymes de l'Afrique antique », Actes du $21^{e}$ Congrès international des Orientalistes, Paris (1948).

GALAND L., 1949 - « La formation des ethniques dans l'Afrique du Nord romaine (problèmes de méthode et observations) ", Actes et mémoires $d u 3^{e}$ Congrès international de toponymie et d'anthroponymie (Bruxelles, 1949), vol. III, Louvain, 1951, p. 778-786.

GALAND L., 1950 - « Onomastique de l'Afrique Ancienne », Rev. Intern. d'Onomastique, 1, p. 67-69.

GALAND L., 1950 - Rubrique « Afrique du Nord », Rev. Intern. d'Onomastique, I (et suiv.), p. 75-76.

GALAND L., 1974 - « Défini, Indéfini, Non-défini : les supports de détermination en touareg », BSLP, LXIX/1, p. 205-224.

GALAND L., 1989 - «"T(h)” in Libyan and Canarian place-names », Almovaren, XX/1, p. 32-41.

GALAND L. \& MEEUSSEN A. E., 1953 et sq. - « Afrique-Africa », Onoma, Louvain, à partir du nº IV, 1953.

HART D.M., 1960 - "Tribal and Place names among the Arabo-berbers of the north-western

Morocco", Hespéris-Tamuda, 1/3.

IBN KHALDOUN - Histoire des Berbères..., Paris, Geuthner.

JONGELING K., 1984 - Names in Neopunic Inscriptions, Université de Groningue, 1984.

JONGELING K., 1994 - North African Names from Latin Sources, Leiden.

LAOUST E., 1939/1942 - « Contribution à une étude de la toponymie du Haut-Atlas», Rev. des Etudes Islamiques, 2, et Contribution à une étude de la toponymie du Haut Atlas, Paris, Geuthner, 1942.

LASSERE J.-M., voir la bibliographie de la notice suivante (017).

LERICHE A., 1958/1959 - « Note sur la langue berbère de Mauritanie. Au sujet de la filiation et des noms de tribus », Bull, de l'Inst. Français d'Afr. Noire, XX, 1958, et African Abstracts, X, 1959.

LEWICKI T., 1955 - Etudes Ibadhites nord-africaines, Varsovie, Polska Akad. Nauk., Komitet Orientalityczny, Prace Orientalistvczne.

LEWICKI T., 1961 - “Ibaditica, 1 : Tasmiya Šuyûả Nafûsa)”, Rocznik Orientalistyezny, XXV, 2, 1961.

LEWICKI T., 1966/1967 - « Sur le nom de Dieu chez les Berbères médiévaux », Folia Orientalia, 8.

MERCIER G., 1897 - «Etude sur la toponymie berbère de la région des Aurès », Actes du XI Congr. Intern. des Orientalistes.

MERCIER G., 1924 - « La langue libyenne et la toponymie antique de l'Afrique du Nord », Journal Asiatique. 
MONTEIL V., 1950/1951 - « La part du berbère dans la toponymie du Sahara maure », Notes africaines, 1-5. $21 ; 1950$ et Actes du Ille Congr. de Toponymie et d'Anthroponymie, 1951.

NAÏT-ZERRAD K., 1983 - L'officiel des prénoms berbères, Paris, L'Harmattan.

PELLEGRIN A., 1953 - Essai sur les noms de lieux d'Algérie et de Tunisie, Tunis.

PICARD A., 1948 - «Compléments à la toponymie berbère », Onomastica.

POTIRON G., 1956 - Tribus (Liste des tribus berbères), Mémoire de D.E.S., Université d'Alger.

ROUX A., 1951 - «Quelques remarques sur la formation des noms de tribus chez les berbérophones du Maroc ", Actes et Mém. Du Ille Congr. de Toponymie et d'Anthroponymie.

RÖSSLER O., 1958 - «Die Sprache Numidiens », Sybaris, Wiesbaden (Festschrift Hans Krahe), p. $94-120$.

WöLFEL D.J., 1951 - « Die Gottesnamen der Libyer und der Berber », Sprache, II, 3.

\section{NOTES}

1. On retient ici la forme primitive du masculin $w$, qui est celle donnée par le corpus libyque, mais

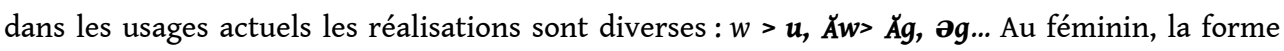
fondamentale est wələt, wəlt > ult, "fille de" (cf. notice M67b « Matriarcat », EB XXXI).

\section{INDEX}

Mots-clés : Antiquité, Linguistique, Moyen Âge, Onomastique 